\title{
Effects of Hall Currents and Radiation on Unsteady MHD Flow Past a Heated Moving Vertical Plate
}

\author{
S. Das ${ }^{1 \dagger}$, S. K. Guchhait ${ }^{2}$ and R. N. Jana ${ }^{3}$ \\ ${ }^{1}$ Department of Mathematics, University of Gour Bnaga, Malda 732 103, West Bengal, India \\ ${ }^{2,3}$ Department of Applied Mathematics, Vidyasagar University, Midnapore 721 102, West Bengal, India \\ †Corresponding Author Email: jana261171@yahoo.co.in
}

(Received September 12, 2013; accepted December 3, 2013)

\begin{abstract}
The effects of Hall currents and radiation on MHD flow of a viscous incompressible electrically conducting fluid past a moving vertical plate with variable temperature in the presence of a uniform transverse magnetic field have been studied. The governing equations are solved analytically using the Laplace transform technique. Effects of the physical parameters on the velocity (both primary and secondary) profiles and temperature distribution are shown graphically and the results are discussed.
\end{abstract}

Keywords: Hall Currents, MHD flow, Radiation, Heated vertical plate, Prandtl Number and Grashof Number.

\section{NOMENCLATURE}

$\begin{array}{cl}B_{x}, B_{y}, B_{z} & \text { magnetic field components } \\ \vec{B} & \text { magnetic field vector } \\ c_{p} & \text { specific heat at constant pressure } \\ E_{x}, E_{y} & \text { electric field components } \\ \vec{E} & \text { electric field vector } \\ e_{\lambda_{p}} & \text { planck's function } \\ F & \text { complex fluid velocity defined by (26) } \\ g & \text { acceleration due to gravity } \\ G r & \text { Grashof number } \\ i=\sqrt{-1} & \text { complex quantity } \\ \vec{j} & \text { current density vector } \\ j_{x}, j_{y}, j_{z} & \text { current density components } \\ K_{\lambda} & \text { absorption coefficient } \\ k & \text { thermal conductivity }\end{array}$

$\begin{array}{cl}m & \text { Hall parameter } \\ M^{2} & \text { magnetic parameter } \\ p & \text { fluid pressure } \\ \operatorname{Pr} & \text { Prandtl number } \\ q_{r} & \text { radiative heat flux } \\ \vec{q} & \text { velocity field vector } \\ R & \text { radiation parameter } \\ T & \text { fluid temperature } \\ T_{w} & \text { plate temperature } \\ T_{\infty} & \text { free stream temperature } \\ U_{0} & \text { uniform plate velocity } \\ u, v & \text { fluid velocity components } \\ u_{1}, v_{1} & \text { dimensionless velocity components } \\ (x, y) & \text { cartesian co-ordinates }\end{array}$

Greek symbols

$\begin{array}{cl}\rho_{0} & \text { reference fluid density } \\ \sigma & \text { conductivity of fluid } \\ \omega_{e} & \text { electron frequency } \\ \tau & \text { non-dimensional time }\end{array}$


$\theta$

$\rho$

dimensionless fluid temperature fluid density

\section{INTRODUCTION}

The problems of magnetohydrodynamic flow and heat transfer have drawn attention of many researchers due to the significant effects of magnetic field on the boundary layer flow control and on the performance of many systems using electrically conducting fluid. In addition, this type of fluid flow finds application in MHD power generation, MHD pumps, flow meters and accelerators, plasma studies, nuclear reactors using liquid metal coolants and geothermal energy extraction. In general, Hall effect devices produce a very low signal level and thus require amplification. While suitable for laboratory instruments, the vacuum tube amplifiers available in the first half of the 20th century were too expensive, power consuming, and unreliable for everyday applications. It was only with the development of the low cost integrated circuit that the Hall effect sensor became suitable for mass applications. These are readily available from a number of different manufacturers, and may be used in various sensors such as rotating speed sensors (bicycle wheels, gear-teeth, automotive speedometers, and electronic ignition systems), fluid flow sensors, current sensors, and pressure sensors. Moreover, the most common application of thermal radiation is a microwave. The thermal radiation effects, on free convection flow are important in context of space technology and in certain applications involving, heat storage in aquifiers and gasification of oil. In the case of gasification large temperature gradients exists in the neighborhood of combustion, due to radiation. These type of problems are also extended in the case of magnetohydrodynamics and if the strength of magnetic field is strong, then the effect of Hall currents cannot be neglected.

The combined effects of Hall currents and radiation on the magnetohydrodynamic flows continue to attract the attention of engineering science and applied mathematics researchers owing to extensive applications of such flows in the context of ionized aerodynamics (Soundalgekar and Takhar (1977)), nuclear energy systems control (Soundalgekar et al. (1981)), improved designs in aerospace MHD energy systems (Ram and Takhar (1994)), manufacture of advanced aerospace materials etc (Takhar and Nath (1998)). Both analytical and computational solutions have been presented to a wide spectrum of problems. Helliwell and Mosa (1979) reported on thermal radiation effects in buoyancy-driven hydromagnetic flow in a horizontal channel flow with an axial temperature gradient in the presence of Joule and viscous heating. The Hall current and surface temperature oscillations effects on natural convection magnetohydrodynamic heat-generating flow were considered by Takhar and Ram (1991). Alagoa et al. (1998) studied magnetohydrodynamic opticallytransparent free-convection flow, with radiative

$$
\begin{array}{cl}
\tau_{e} & \text { electron collision time } \\
\tau_{x}, \tau_{y} & \text { shear stresses at the plate }
\end{array}
$$

heat transfer in porous media with time-dependent suction using an asymptotic approximation, showing that thermal radiation exerts a significant effect on the flow dynamics. The magnetohydrodynamic free convection heat and mass transfer of a heat generating fluid past an impulsively started infinite vertical porous plate with Hall current and radiation absorption was studied by Kinyanjui (2001). Aboeldahab and Elbarbary (2001) have presented the effects of Hall currents on magnetohydrodynamic free convection flow past a semi-infinite vertical plate with mass transfer. Cookey et al. (2003) investigated the influence of viscous dissipation and radiation on unsteady MHD free convection flow past an infinite heated vertical plate in a porous media with time dependent suction. Aboeldahab and El Aziz (2005) have studied the viscous dissipation and Joule heating effects on MHD free convection from a vertical plate with power-law variation in surface temperature in the presence of Hall and ion-slip currents. The thermal radiation interaction with unsteady MHD flow past a vertical porous plate immersed in a porous medium was investigated by Samad and Rahman (2006). Chaudhary and Jain (2007) studied the behaviours of unsteady hydromagnetic flow of a visco-elastic fluid from a radiative vertical porous plate. The effects of thermal radiation and Hall currents on magnetohydrodynamic free-convective flow and mass transfer over a stretching sheet with variable viscosity in the presence of heat generation/absorption were investigated by Shit and Haldar (2010). Israel-Cookey (2010) studied the MHD oscillatory Couette flow of a radiating viscous fluid in a porous medium with periodic wall temperature. The effects of thermal radiation, Hall currents, Soret and Dufour on MHD flow by mixed convection over a vertical surface in a porous medium where described by Shateyi (2010). Aurangzaib and Sharidan Shafie (2011) studied the effects of Soret and Dufour on unsteady MHD flow by mixed convection over a vertical surface in a porous medium with internal heat generation, chemical reaction and Hall current. Singh and Pathak (2012) discussed the effects of rotation and Hall current on mixed convection MHD flow through a porous medium in a vertical channel in the presence of thermal radiation. Jain and Singh (2012) have observed Hall and thermal radiative effects on an unsteady rotating free convection slip flow. Recently, Chaudhary et al. (2013) have presented the effects of Hall current and thermal radiation on an unsteady free convection slip flow along a vertical plate embedded in a porous medium with constant heat and mass flux. Seth et al. (2013) have examined the effects of thermal radiation and rotation on unsteady hydromagnetic free convection flow past an impulsively moving vertical plate with ramped temperature in a porous medium. The unsteady MHD radiative and chemically reactive free convection flow near a moving vertical plate in porous medium has been presented by Reddy et al. 
(2013). Choudhury and Hazarika (2013) have studied the effects of variable viscosity and thermal conductivity on MHD oscillatory free convective flow past a vertical plate in slip flow regime with variable suction and periodic plate temperature.

In the present paper, we study the effects of Hall currents and radiation on MHD flow of a viscous incompressible electrically conducting fluid past a moving vertical plate with variable temperature in the presence of a uniform transverse magnetic filed. The governing equations are solved by the Laplace transform technique to obtain the analytical results for velocity, temperature, rate of heat transfer and shear stresses. The effects of physical parameters on the velocity, temperature and rate of heat transfer and shear stresses are analyzed in detail.

\section{FORMULATION OF THE PROBLEM AND ITS SOLUTION}

Consider the viscous incompressible electrically conducting fluid past a moving vertical plate with variable plate temperature. Choose a Cartesian coordinates system with $x$-axis along the plate in the direction of the flow, the $y$-axis is normal to the plate and the $z$-axis perpendicular to $x y$-plane [see Fig.1.] Initially, at time $t \leq 0$, both the plate and the fluid are assumed to be at the same temperature $T_{\infty}$ and stationary. At time $t>0$, the plate at $z=0$ starts moving in its own plane with a uniform velocity $U_{0}$ and is heated with temperature $T_{\infty}+\left(T_{w}-T_{\infty}\right) \frac{t}{t_{0}}$. A uniform magnetic field of strength $B_{0}$ is imposed perpendicular to the plate. It is also assumed that the radiative heat flux in the $x$ -direction is negligible as compared to that in the $z$ - direction. As the plates are infinitely long, the velocity and temperature fields are functions of $z$ and $t$ only. The equation of continuity then gives $w=0$ everywhere in the fluid where $\vec{q}=(u, v, w)$.

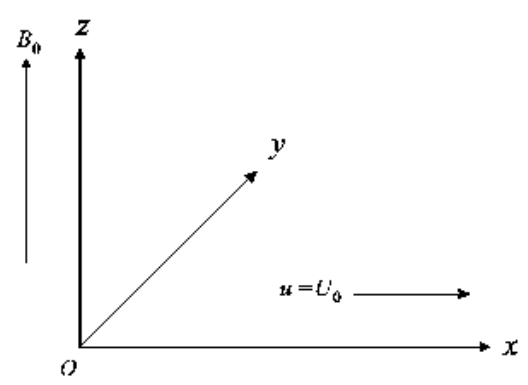

Fig. 1. Geometry of the problem

The Boussinesq approximation is assumed to hold and for the evaluation of the gravitational body force, the density is assumed to be dependent on the temperature according to the equation of state

$$
\rho=\rho_{0}\left[1-\beta\left(T-T_{\infty}\right)\right],
$$

where $T$ is the fluid temperature, $\rho$ the fluid density, $\beta$ the coefficient of thermal expansion and $\rho_{0}$ the reference fluid density.

Using Boussinesq approximation, the momentum equations of motion along $x$ and $y$-directions are

$$
\begin{aligned}
& \frac{\partial u}{\partial t}=-\frac{1}{\rho} \frac{\partial p}{\partial x}+v \frac{\partial^{2} u}{\partial z^{2}}+g \beta\left(T-T_{\infty}\right)+\frac{B_{0}}{\rho} j_{y}, \\
& \frac{\partial v}{\partial t}=-\frac{1}{\rho} \frac{\partial p}{\partial y}+v \frac{\partial^{2} v}{\partial z^{2}}-\frac{B_{0}}{\rho} j_{x},
\end{aligned}
$$

where $v$ and $p$ are respectively the kinematic viscosity and the modified fluid pressure.

Neglecting ion-slip and thermoelectric effects, the generalised Ohm's law for partially ionized gas is (see Cowling (1957))

$\vec{j}+\frac{\omega_{e} \tau_{e}}{B_{0}}(\vec{j} \times \vec{B})=\sigma(\vec{E}+\vec{q} \times \vec{B})$,

where $\vec{B}, \vec{E}, \vec{q}, \vec{j}, \sigma, \omega_{e}$ and $\tau_{e}$ are respectively, the magnetic field vector, the electric field vector, the fluid velocity vector, the current density vector, the conductivity of the fluid, the cyclotron frequency and the electron collision time. We shall assume that the magnetic Reynolds number for the flow is small so that the induced magnetic field can be neglected. This assumption is justified since the magnetic Reynolds number is generally very small for partially ionized gases (Shereliff (1965)). The solenoidal relation $\nabla \cdot \vec{B}=0$ for the magnetic field gives $B_{z}=B_{0}=$ constant everywhere in the fluid where $\vec{B} \equiv\left(0,0, B_{0}\right)$. The equation of the conservation of the charge $\nabla \cdot \vec{j}=0$ gives $j_{z}=$ constant. This constant is zero since $j_{z}=0$ at the plate which is electrically nonconducting. Thus $j_{z}=0$ everywhere in the flow. Since the induced magnetic field is neglected, the Maxwell's equation $\nabla \times \vec{E}=-\frac{\partial \vec{B}}{\partial t} \quad$ becomes $\nabla \times \vec{E}=0$ which gives $\frac{\partial E_{x}}{\partial z}=0$ and $\frac{\partial E_{y}}{\partial z}=0$. This implies that $E_{x}=$ constant and $E_{y}=$ constant everywhere in the flow.

In view of the above assumption, Eq.(4) gives

$j_{x}+m j_{y}=\sigma\left(E_{x}+v B_{0}\right)$,

(5)

$j_{y}-m j_{x}=\sigma\left(E_{y}-u B_{0}\right)$,

(6)

where $m=\omega_{e} \tau_{e}$ is the Hall parameter. Solving for $j_{x}$ and $j_{y}$, we get

$j_{x}=\frac{\sigma}{1+m^{2}}\left[\left(E_{x}+v B_{0}\right)-m\left(E_{y}-u B_{0}\right)\right]$,

$j_{y}=\frac{\sigma}{1+m^{2}}\left[m\left(E_{x}+v B_{0}\right)+\left(E_{y}-u B_{0}\right)\right]$.

On the use of Eqs. (7) and (8), Eqs. (2) and (3) become

$$
\begin{aligned}
& \frac{\partial u}{\partial t}=-\frac{1}{\rho} \frac{\partial p}{\partial x}+v \frac{\partial^{2} u}{\partial z^{2}}+g \beta\left(T-T_{\infty}\right) \\
& +\frac{\sigma B_{0}}{\rho\left(1+m^{2}\right)}\left[m\left(E_{x}+v B_{0}\right)+\left(E_{y}-u B_{0}\right)\right],
\end{aligned}
$$


S. Das et al. / JAFM, Vol. 7, No. 4, pp. 683-692, 2014.

$$
\begin{aligned}
& \frac{\partial v}{\partial t}=-\frac{1}{\rho} \frac{\partial p}{\partial y}+v \frac{\partial^{2} v}{\partial z^{2}} \\
& -\frac{\sigma B_{0}}{\rho\left(1+m^{2}\right)}\left[\left(E_{x}+v B_{0}\right)-m\left(E_{y}-u B_{0}\right)\right] .
\end{aligned}
$$

The energy equation is

$\rho c_{p} \frac{\partial T}{\partial t}=k \frac{\partial^{2} T}{\partial y^{2}}-\frac{\partial q_{r}}{\partial y}$,

where $k$ is the thermal conductivity, $c_{p}$ the specific heat at constant pressure and $q_{r}$ the radiative heat flux. The heat due to viscous dissipation is neglected for small velocities in the energy Eq.(11).

The initial and the boundary conditions for velocity and temperature distribution are

$u=0, v=0, T=T_{\infty}$ for all $z$ and $t \leq 0$,

$u=U_{0}, v=0, T=T_{\infty}+\left(T_{w}-T_{\infty}\right) \frac{t}{t_{0}}$

at $z=0$ for $t>0$,

$u \rightarrow 0, v \rightarrow 0, T \rightarrow T_{\infty}$ as $z \rightarrow \infty$ for $t>0$.

It has been shown by Cogley et al. (1968) that in the optically thin limit for a non-gray gas near equilibrium, the following relation holds

$\frac{\partial q_{r}}{\partial y}=4\left(T-T_{\infty}\right) \int_{0}^{\infty} K_{\lambda_{w}}\left(\frac{\partial e_{\lambda p}}{\partial T}\right)_{w} d \lambda$

where $K_{\lambda}$ is the absorption coefficient, $\lambda$ is the wave length, $e_{\lambda p}$ is the Planck's function and subscript ' $w$ ' indicates that all quantities have been evaluated at the temperature $T_{\infty}$ which is the temperature of the plate at time $t \leq 0$. Thus our study is limited to small difference of plate temperature to the fluid temperature.

On the use of the Eq.(13), the Eq. (11) becomes

$\rho c_{p} \frac{\partial T}{\partial t}=k \frac{\partial^{2} T}{\partial y^{2}}-4\left(T-T_{\infty}\right) I$,

where

$I=\int_{0}^{\infty} K_{\lambda_{w}}\left(\frac{\partial e_{\lambda p}}{\partial T}\right)_{w} d \lambda$.

On the use of infinity conditions, Eqs (9) and (10) yield

$-\frac{1}{\rho} \frac{\partial p}{\partial x}+\frac{\sigma B_{0}}{\rho}\left(m E_{x}+E_{y}\right)=0$,
$-\frac{1}{\rho} \frac{\partial p}{\partial y}-\frac{\sigma B_{0}}{\rho}\left(E_{x}-m E_{y}\right)=0$.

On the use of (16) and (17), Eqs.(9) and (10) become

$$
\begin{aligned}
& \frac{\partial u}{\partial t}=g \beta\left(T-T_{\infty}\right)+v \frac{\partial^{2} u}{\partial z^{2}}-\frac{\sigma B_{0}^{2}}{\rho}(m v-u), \\
& \frac{\partial v}{\partial t}=v \frac{\partial^{2} u}{\partial z^{2}}-\frac{\sigma B_{0}^{2}}{\rho}(v+m u) .
\end{aligned}
$$

Introducing non-dimensionless variables

$$
\eta=\frac{z U_{0}}{v}, \tau=\frac{v t}{U_{0}}, u_{1}=\frac{u}{U_{0}}, v_{1}=\frac{v}{U_{0}}, \theta=\frac{T-T_{\infty}}{T_{w}-T_{\infty}},
$$

equations (18), (19) and (14) become

$\frac{\partial u_{1}}{\partial \tau}=\frac{\partial^{2} u_{1}}{\partial \eta^{2}}-M^{2}\left(u_{1}-m v_{1}\right)+G r \theta$,

$\frac{\partial v_{1}}{\partial \tau}=\frac{\partial^{2} v_{1}}{\partial \eta^{2}}-M^{2}\left(v_{1}+m u_{1}\right)$,

$\operatorname{Pr} \frac{\partial \theta}{\partial \tau}=\frac{\partial^{2} \theta}{\partial \eta^{2}}-R \theta$

where $M^{2}=\frac{\sigma B_{0}^{2} v}{\rho U_{0}^{2}}$ is the magnetic parameter, $R=\frac{4 I h^{2}}{k} \quad$ the radiation parameter, $G r=\frac{g \beta v\left(T_{w}-T_{\infty}\right)}{U_{0}^{3}}$ the Grashof number and $\operatorname{Pr}=\frac{\rho v c_{p}}{k}$ the Prandtl number.

The corresponding initial and boundary conditions for $u_{1}$ and $\theta$ are

$$
\begin{aligned}
& u_{1}=0, v_{1}=0, \theta=0 \text { for all } \eta \text { and } \tau \leq 0, \\
& u_{1}=1, v_{1}=0, \theta=\tau \text { at } \eta=0 \text { for } \tau>0, \\
& u_{1} \rightarrow 0, v_{1} \rightarrow 0, \theta \rightarrow 0 \text { as } \eta \rightarrow 0 \text { for } \tau>0 .
\end{aligned}
$$

Combining Eqs. (21) and (22), we have

$$
\frac{\partial F}{\partial \tau}=\frac{\partial^{2} F}{\partial \eta^{2}}-\frac{M^{2}(1+i m)}{1+m^{2}}+G r \theta,
$$

where

$F=u_{1}+i v_{1}, i=\sqrt{-1}$.

The corresponding initial and boundary conditions for $F$ and $\theta$ are

$$
\begin{aligned}
& F=0, \theta=0 \text { for all } \eta \text { and } \tau \leq 0, \\
& F=1, \theta=\tau \text { at } \eta=0 \text { for } \tau>0, \\
& F \rightarrow 0, \theta \rightarrow 0 \text { as } \eta \rightarrow 0 \text { for } \tau>0 .
\end{aligned}
$$

Taking Laplace transformation and on using initial conditions for $F(\eta, \tau)$ and $\theta(\eta, \tau)$, Eqs.(25) and (23) become

$$
\begin{aligned}
& \frac{d^{2} \bar{F}}{d \eta^{2}}-\left[s+\frac{M^{2}(1+i m)}{1+m^{2}}\right] \bar{F}=-G r \bar{\theta}, \\
& \frac{d^{2} \bar{\theta}}{d \eta^{2}}-(s \operatorname{Pr}+R) \bar{\theta}=0,
\end{aligned}
$$

where

$$
\begin{aligned}
& \bar{F}(\eta, s)=\int_{0}^{\infty} F(\eta, \tau) e^{-s \tau} d \tau \\
& \text { and } \bar{\theta}(\eta, s)=\int_{0}^{\infty} \theta(\eta, \tau) e^{-s \tau} d \tau .
\end{aligned}
$$

The corresponding boundary conditions for $\bar{F}$ and $\bar{\theta}$ are 
S. Das et al. / JAFM, Vol. 7, No. 4, pp. 683-692, 2014.

$$
\begin{aligned}
& \bar{F}(0, s)=\frac{1}{s}, \bar{\theta}(0, s)=\frac{1}{s^{2}}, \\
& \bar{F} \rightarrow 0, \bar{\theta} \rightarrow 0 \text { as } \eta \rightarrow 0 .
\end{aligned}
$$

The solution of Eqs.(29) and (28) subject to the boundary conditions (31) are easily obtained and are given by

$$
\begin{aligned}
& \bar{\theta}(\eta, s)= \begin{cases}\frac{1}{s^{2}} e^{-\sqrt{s P r+R} \eta} & \text { for } \operatorname{Pr} \neq 1 \\
\frac{1}{s^{2}} e^{-\sqrt{s+R} \eta} & \text { for } \operatorname{Pr}=1,\end{cases} \\
& \bar{F}(\eta, s)= \begin{cases}\left(\frac{1}{s}+\frac{G_{1}}{b^{2} s}+\frac{G_{1}}{b s^{2}}\right) e^{-\sqrt{s+r^{2}} \eta} \\
-G_{1}\left(\frac{1}{b^{2} s}+\frac{1}{b s^{2}}\right) e^{-\sqrt{\operatorname{Pr}(s+a)} \eta} \\
+\frac{G_{1}}{b^{2}(s-b)} e^{-\sqrt{\operatorname{Pr}(s+a)} \eta} \\
-\frac{G_{1}}{b^{2}(s-b)} e^{-\sqrt{s+r^{2}} \eta} \text { for } \operatorname{Pr} \neq 1 \\
\left(\frac{1}{s}-\frac{G_{2}}{s^{2}}\right) e^{-\sqrt{s+r^{2}} \eta}+\frac{G_{2}}{s^{2}} e^{-\sqrt{s+R} \eta} \text { for } \operatorname{Pr}=1\end{cases}
\end{aligned}
$$

where $\quad a=\frac{R}{P r}, G_{1}=\frac{G r}{1-P r}, \quad G_{2}=\frac{G r}{r^{2}-R} \quad$ and

$$
r^{2}=\frac{M^{2}(1+i m)}{1+m^{2}} .
$$

The inverse transforms of Eqs.(32) and (33) give the temperature and the velocity field distributions as

$$
\begin{aligned}
& \theta(\eta, \tau)= \\
& \left\{\begin{array}{l}
\frac{1}{2}\left[\left(\tau+\frac{\eta \sqrt{P r}}{2 \sqrt{a}}\right) e^{\eta \sqrt{a P r}} \operatorname{erfc}\left(\frac{\eta \sqrt{P r}}{2 \sqrt{\tau}}+\sqrt{a \tau}\right)\right. \\
\left.+\left(\tau-\frac{\eta \sqrt{P r}}{2 \sqrt{a}}\right) e^{-\eta \sqrt{a P r}} \operatorname{erfc}\left(\frac{\eta \sqrt{P r}}{2 \sqrt{\tau}}-\sqrt{a \tau}\right)\right] \text { for } \operatorname{Pr} \neq 1 \\
\frac{1}{2}\left[\left(\tau+\frac{\eta}{2 \sqrt{a}}\right) e^{\eta \sqrt{a}} \operatorname{erfc}\left(\frac{\eta}{2 \sqrt{\tau}}+\sqrt{a \tau}\right)\right. \\
\left.+\left(\tau-\frac{\eta}{2 \sqrt{a}}\right) e^{-\eta \sqrt{a}} \operatorname{erfc}\left(\frac{\eta}{2 \sqrt{\tau}}-\sqrt{a \tau}\right)\right] \text { for } \operatorname{Pr}=1
\end{array}\right.
\end{aligned}
$$

$$
\begin{aligned}
& F(\eta, \tau)= \\
& \left\{\begin{array}{l}
\frac{1}{2}\left(1+\frac{G_{1}}{b^{2}}+\frac{G_{1} \tau}{b}+\frac{G_{1} \eta}{2 b r}\right) e^{r \eta} \operatorname{erfc}\left(\frac{\eta}{2 \sqrt{\tau}}+r \sqrt{\tau}\right) \\
+\frac{1}{2}\left(1+\frac{G_{1}}{b^{2}}+\frac{G_{1} \tau}{b}-\frac{G_{1} \eta}{2 b r}\right) e^{-r \eta} \operatorname{erfc}\left(\frac{\eta}{2 \sqrt{\tau}}-r \sqrt{\tau}\right)
\end{array}\right. \\
& -\frac{G_{1}}{2 b}\left(\tau+\frac{\eta \sqrt{P r}}{2 \sqrt{a}}+\frac{1}{b}\right) e^{\eta \sqrt{a P r}} \operatorname{erfc}\left(\frac{\eta \sqrt{P r}}{2 \sqrt{\tau}}+\sqrt{a \tau}\right) \\
& -\frac{G_{1}}{2 b}\left(\tau-\frac{\eta \sqrt{P r}}{2 \sqrt{a}}+\frac{1}{b}\right) e^{-\eta \sqrt{a a_{r}}} \operatorname{erfc}\left(\frac{\eta \sqrt{P r}}{2 \sqrt{\tau}}-\sqrt{a \tau}\right) \\
& -\frac{G_{1} e^{b \tau}}{2 b^{2}}\left[e^{\eta \sqrt{r^{2}+b}} \operatorname{erfc}\left(\frac{\eta}{2 \sqrt{\tau}}+\sqrt{\left(r^{2}+b\right) \tau}\right)\right. \\
& \left.+e^{-\eta \sqrt{r^{2}+b}} \operatorname{erfc}\left(\frac{\eta}{2 \sqrt{\tau}}-\sqrt{\left(r^{2}+b\right) \tau}\right)\right] \\
& +\frac{G_{G} e^{b \tau}}{2 b^{2}}\left[e^{\eta \sqrt{\operatorname{Pr}(a+b)}} \operatorname{erfc}\left(\frac{\eta \sqrt{\operatorname{Pr}}}{2 \sqrt{\tau}}+\sqrt{(a+b) \tau}\right)\right. \\
& \left.+e^{-\eta \sqrt{P r(a+b)}} \operatorname{erfc}\left(\frac{\eta \sqrt{\operatorname{Pr}}}{2 \sqrt{\tau}}-\sqrt{(a+b) \tau}\right)\right] \text { for } \operatorname{Pr} \neq 1 \\
& \frac{1}{2}\left(1-G_{2} \tau-\frac{G_{2} \eta}{2 r}\right) e^{r \eta} \operatorname{erfc}\left(\frac{\eta}{2 \sqrt{\tau}}+r \sqrt{\tau}\right) \\
& +\frac{1}{2}\left(1-G_{2} \tau+\frac{G_{2} \eta}{2 r}\right) e^{-r \eta} \operatorname{erfc}\left(\frac{\eta}{2 \sqrt{\tau}}-r \sqrt{\tau}\right) \\
& +\frac{1}{2} G_{2}\left[\left(\tau+\frac{\eta}{2 \sqrt{R}}\right) e^{\eta \sqrt{\bar{R}}} \operatorname{erfc}\left(\frac{\eta}{2 \sqrt{\tau}}+\sqrt{R \tau}\right)\right. \\
& \left.+\left(\tau-\frac{\eta}{2 \sqrt{R}}\right) e^{-\eta \sqrt{R}} \operatorname{erfc}\left(\frac{\eta}{2 \sqrt{\tau}}-\sqrt{R \tau}\right)\right] \text { for } P r=1
\end{aligned}
$$

where erfc (.) is the complementary error function.

\section{RESULTS AND DISCUSSION}

We have presented the non-dimensional velocity components and temperature distribution for several values of magnetic parameter $M^{2}$, radiation parameter $R$, Hall parameter $m$, Prandtl number $\mathrm{Pr}$, Grashof number $\mathrm{Gr}$ and time $\tau$ in Figs.2 to 10. Figures. 2 to 7 represent the primary velocity $u_{1}$ and secondary velocity $v_{1}$ against $\eta$ for several values of $M^{2}, R, m, P r, G r$ and $\tau$. It is seen from Fig. 2 that the primary velocity $u_{1}$ decreases where as the magnitude of the secondary velocity $v_{1}$ increases with an increase in magnetic parameter $M^{2}$. The application of the transverse magnetic field plays the role of a resistive type force (Lorentz force) similar to drag force (that acts in the opposite direction of the fluid motion) which tends to resist the flow thereby reducing its velocity. The effect of radiation parameter on the velocity field is shown in Fig.3. It is observed that both the primary velocity $u_{1}$ and the magnitude of the secondary velocity $v_{1}$ decrease with an increase in radiation parameter $R$. This means that there is a fall in velocity in the presence of radiation. It is seen from Fig. 4 that both the primary velocity $u_{1}$ and the magnitude of the secondary velocity $v_{1}$ increase with an increase in Hall parameter $m$. 
Fig.5 illustrates that both the primary velocity $u_{1}$ and the magnitude of the secondary velocity $v_{1}$ decrease with an increase in Prandtl number $\mathrm{Pr}$. Physically, this is true because the increase in the Prandtl number is due to increase in the viscosity of the fluid which makes the fluid thick and hence causes a decrease in the velocity of the fluid. It is observed from Fig.6 that an increase in $G r$ leads to rise in the both values of the primary velocity $u_{1}$ and the magnitude of the secondary velocity $v_{1}$. An increase in Grashof number leads to an increase in velocity, this is because, increase in Grashof number means more heating and less density. It is seen from Fig.7 that the both the primary velocity $u_{1}$ and the magnitude of the secondary velocity $v_{1}$ increase with an increase in time $\tau$. It is seen from Fig. 8 that the temperature $\theta$ decreases with an increase in radiation parameter $R$. This result qualitatively agrees with expectations, since the effect of radiation is to decrease the rate of energy transport to the fluid, thereby decreasing the temperature of the fluid. Fig.9 shows that the temperature $\theta$ decreases with an increase in Prandtl number $\mathrm{Pr}$. This implies that an increase in Prandtl number leads to fall the thermal boundary layer flow. This is because fluids with large $\mathrm{Pr}$ have low thermal diffusivity which causes low heat penetration resulting in reduced thermal boundary layer. Fig.10 reveals that the temperature $\theta$ increases with an increase in time $\tau$. The trend shows that the temperature increases with increasing time.

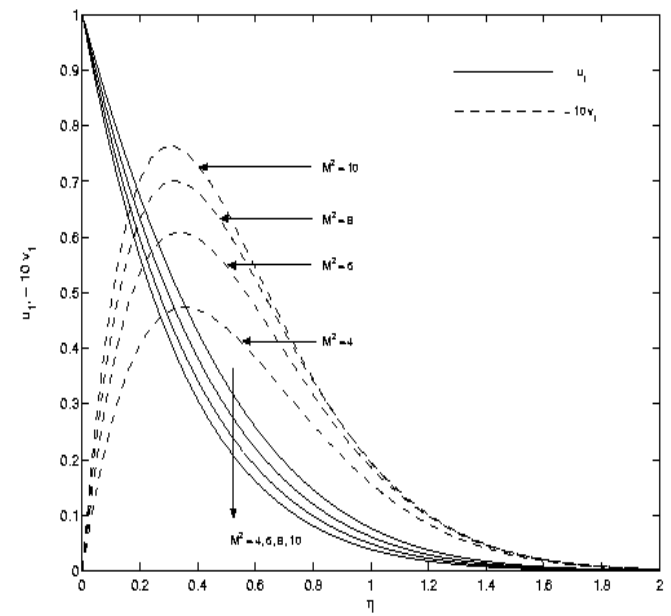

Fig. 2. Velocity profiles for $M^{2}$ when $R=4$, $m=0.5, P r=0.71, G r=5$ and $\tau=0.5$

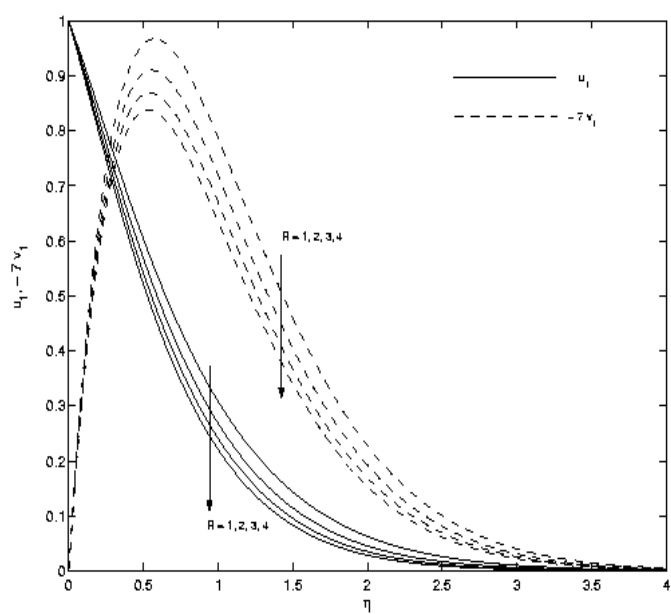

Fig. 3. Velocity profiles for $R$ when $M^{2}=5$, $G r=5, P r=0.71, m=0.5$ and $\tau=0.5$

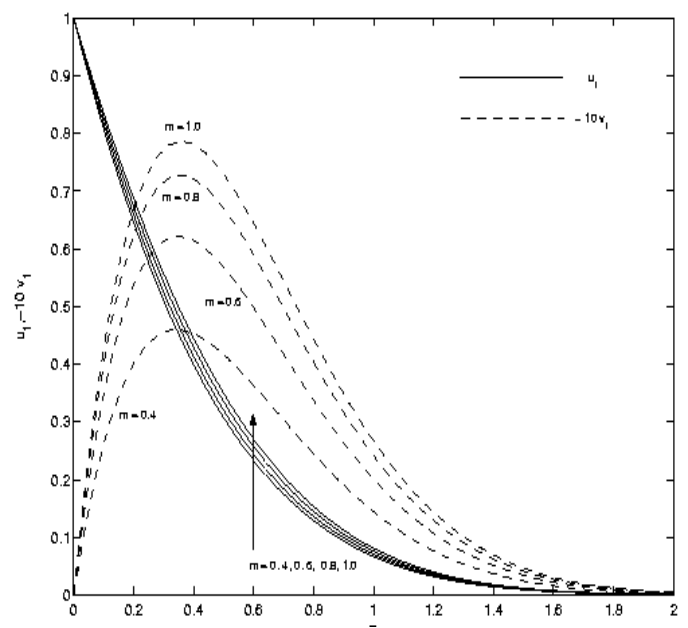

Fig. 4. Velocity profiles for $m$ when $M^{2}=5$, $R=4, G r=5, P r=0.71$ and $\tau=0.5$

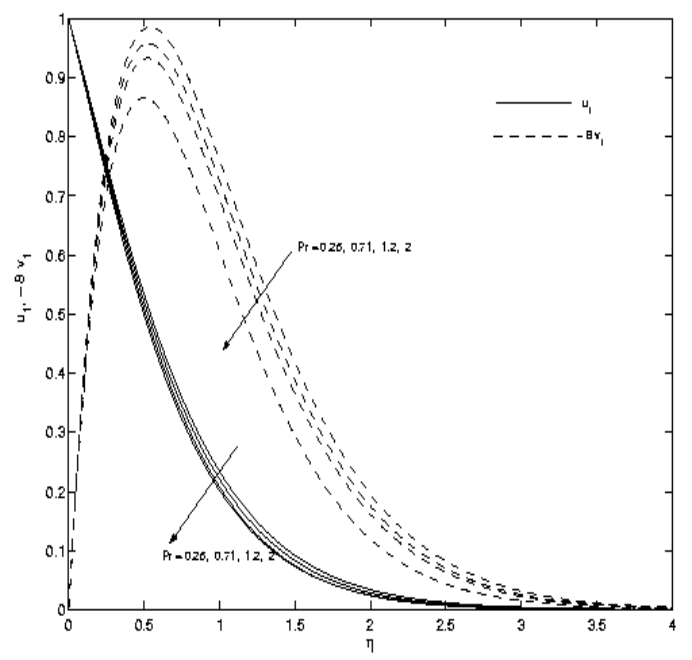

Fig. 5. Velocity profiles for $\operatorname{Pr}$ when $M^{2}=5$, $R=4, G r=5, m=0.5$ and $\tau=0.5$ 


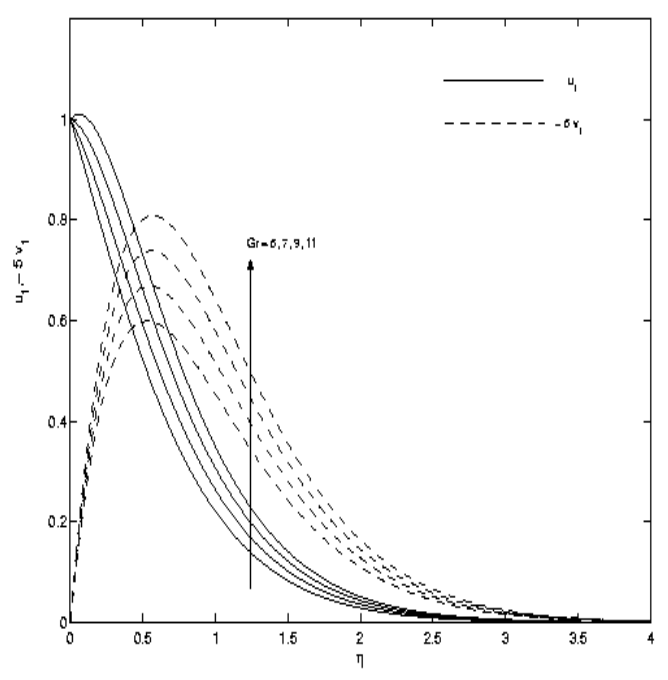

Fig. 6. Velocity profiles for $G r$ when $M^{2}=5$, $R=4, P r=0.71, m=0.5$ and $\tau=0.5$

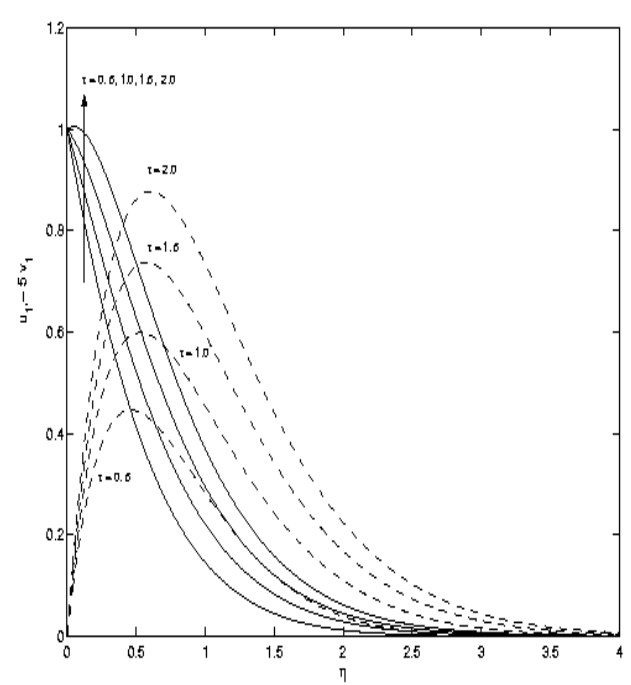

Fig. 7. Velocity profiles for $\tau$ when $M^{2}=5$, $R=4, G r=5, m=0.5$ and $P r=0.71$

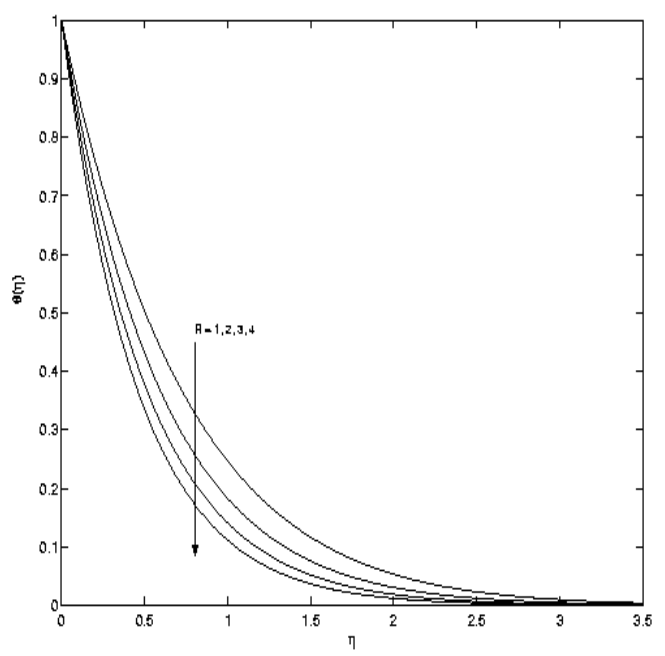

Fig. 8. Temperature profiles for $R$ when $\operatorname{Pr}=0.71$ and $\tau=0.5$

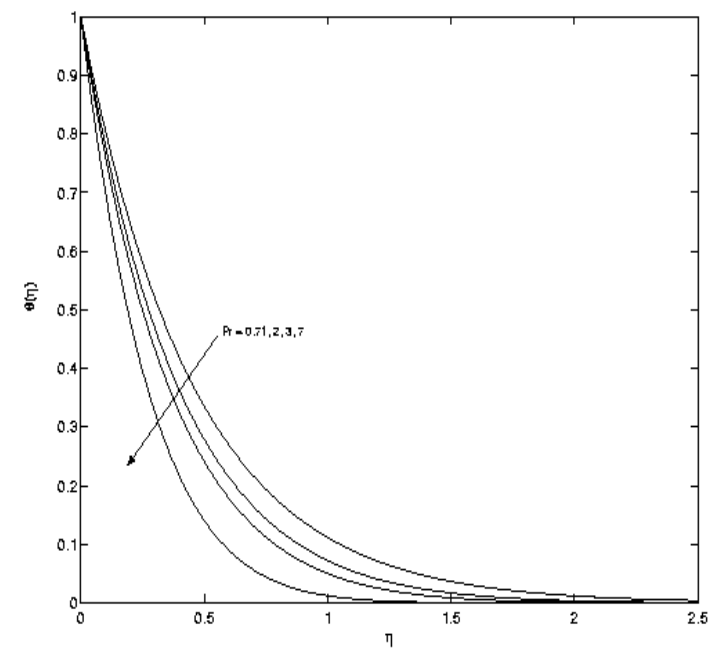

Fig. 9. Temperature profiles for $\operatorname{Pr}$ when $R=4$ and $\tau=0.2$

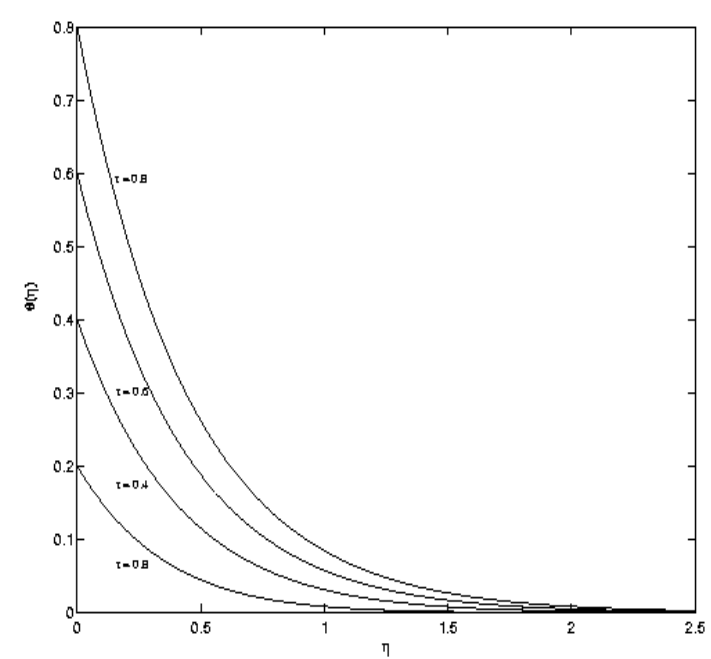

Fig. 10. Temperature profiles for $\tau$ when

$$
\operatorname{Pr}=0.71 \text { and } R=4
$$

The rate of heat transfer at the moving plate $\eta=0$ is given by

$$
-\left(\frac{\partial \theta}{\partial \eta}\right)_{\eta=0}= \begin{cases}+\sqrt{\frac{\tau P r}{\pi}} e^{-a \tau} & \text { for } \operatorname{Pr} \neq 1 \\ \left(\tau \sqrt{a}+\frac{1}{2 \sqrt{a}}\right) & \operatorname{erf}\left(\sqrt{\frac{P r}{a \tau}}\right) \\ \operatorname{erf}(\sqrt{a \tau}) \\ +\sqrt{\frac{\tau}{\pi}} e^{-a \tau} & \text { for } \operatorname{Pr}=1\end{cases}
$$

Numerical results of the rate of heat transfer $-\left(\frac{\partial \theta}{\partial \eta}\right)_{\eta=0}$ at the moving plate $\eta=0$ against the radiation parameter $R$ are presented in the Table 1 for several values of radiation parameter $R$, Prandtl number $\operatorname{Pr}$ and time $\tau$. Table 1 shows that for the fixed value of radiation parameter $R$, the 
rate of heat transfer $-\left(\frac{\partial \theta}{\partial \eta}\right)_{\eta=0}$ increases with an increase in either Prandtl number $\operatorname{Pr}$ or time $\tau$. This may be explained by the fact that frictional forces become dominant with increasing values of $\operatorname{Pr}$ and hence yield greater heat transfer rates. Further, it is seen that for fixed values of $\operatorname{Pr}$ and $\tau$
, the rate of heat transfer $-\left(\frac{\partial \theta}{\partial \eta}\right)_{\eta=0}$ increases with an increase in radiation parameter $R$.

From the physical point of view, it is necessary to know the shear stress at the moving plate. The nondimensional shear stress at the plate $\eta=0$ is

$$
\text { Table } 1 \text { Rate of heat transfer }-\left(\frac{\partial \theta}{\partial \eta}\right)_{\eta=0} \text { at the plate } \eta=0
$$

\begin{tabular}{|c|c|c|c|c|c|c|c|c|}
\hline & \multicolumn{3}{|c|}{$P r$} & \multicolumn{4}{c|}{$\tau$} \\
\hline$R$ & 0.71 & 2 & 3 & 7 & 0.2 & 0.4 & 0.6 & 0.8 \\
\hline 2 & 0.78575 & 0.93166 & 1.14519 & 1.87743 & 0.29814 & 0.60353 & 0.98504 & 1.42837 \\
4 & 0.99997 & 0.91905 & 1.05290 & 1.70618 & 0.33859 & 0.75278 & 1.27002 & 1.87238 \\
6 & 1.16851 & 0.96373 & 1.03091 & 1.58148 & 0.37827 & 0.87221 & 1.49389 & 2.22458 \\
8 & 1.31126 & 1.02273 & 1.04219 & 1.49154 & 0.41138 & 0.97210 & 1.68518 & 2.52734 \\
\hline
\end{tabular}

$$
\begin{aligned}
& \tau_{x}+i \tau_{y}=\left(\frac{\partial F}{\partial \eta}\right)_{\eta=0} \\
& -\left[\frac{G_{1}}{2 b r}+\left(1+\frac{G_{1}}{b^{2}}+\frac{G_{1} \tau}{b}\right)\right] \operatorname{erf}(r \sqrt{\tau}) \\
& -\frac{1}{\sqrt{\pi \tau}}\left(1+\frac{G_{1}}{b^{2}}+\frac{G_{1} \tau}{b}\right) e^{-r^{2} \eta} \\
& +\left[\frac{G_{1}}{2 b} \sqrt{\frac{\operatorname{Pr}}{a}}+\frac{G_{1}}{b}\left(\tau+\frac{1}{b}\right)\right] \operatorname{erf}(\sqrt{a \tau}) \\
& -\frac{G_{1}}{b} \sqrt{\frac{\operatorname{Pr}}{\pi \tau}}\left(\tau+\frac{1}{b}\right) e^{-a \tau} \\
& +\frac{G_{1} e^{b \tau}}{b^{2}}\left[\sqrt{r^{2}+b} \operatorname{erf}\left(\sqrt{\left(r^{2}+b\right) \tau}\right)\right. \\
& -\sqrt{\operatorname{Pr}(a+b)} \operatorname{erf}(\sqrt{\operatorname{Pr}(a+b) \tau})] \\
& +\frac{G_{1} e^{b \tau}}{2 b^{2} \sqrt{\pi \tau}}\left[e^{-\left(r^{2}+b\right) \tau}-\sqrt{\operatorname{Pr}} e^{-(a+b) \tau}\right] \text { for } \operatorname{Pr} \neq 1 \\
& {\left[\begin{array}{l}
\left.\frac{G_{2}}{2 r}-\left(1-G_{2} \tau\right)\right] \operatorname{erf}(r \sqrt{\tau})-\frac{\left(1-G_{2} \tau\right)}{\sqrt{\pi \tau}} e^{-r^{2} \tau} \\
-G_{2}\left(\frac{1}{2 \sqrt{R}}+\tau \sqrt{R}\right) \operatorname{erf}(\sqrt{R \tau}) \\
-G_{2} \sqrt{\frac{\tau}{\pi}} e^{-R \tau}
\end{array} \text { for } \operatorname{Pr}=1\right.}
\end{aligned}
$$

Numerical results of the shear stresses $\tau_{x}$ due to the primary flow and $\tau_{y}$ due to the secondary flow at the plate $\eta=0$ are presented in Figs. 11 to 14 against Hall parameter $m$ for several values of magnetic parameter $M^{2}$, radiation parameter $R$, Prandtl number $\operatorname{Pr}$ and time $\tau$. Fig.11 illustrates that the absolute value of the shear stress $\tau_{x}$ increases whereas the absolute value of the shear stress $\tau_{y}$ decreases with an increase in magnetic parameter $M^{2}$ for the fixed value of Hall parameter $m$. It is also seen that the absolute value of the shear stress $\tau_{x}$ decreases while the absolute value of the shear stress $\tau_{y}$ increases with an increase in Hall parameter for fixed value of the magnetic parameter $M^{2}$. These results are in agrement with the fact that the primary velocity decreases and the secondary increases with an increase in Hall parameter $m$. It is seen from Fig. 12 that the absolute value of the shear stress $\tau_{x}$ increases whereas the absolute value of the shear stress $\tau_{y}$ decreases with an increase in radiation parameter $R$. Fig. 13 reveals that the absolute value of the shear stress $\tau_{x}$ decreases while the result is reversed for the shear stress $\tau_{y}$ with an increase in Prandtl number $\mathrm{Pr}$. Further, it is revealed from Fig.14 that the absolute value of the shear stress $\tau_{x}$ decreases while the absolute value of the shear stress $\tau_{y}$ increases with an increase in time $\tau$ for the fixed value of Hall parameter $m$.

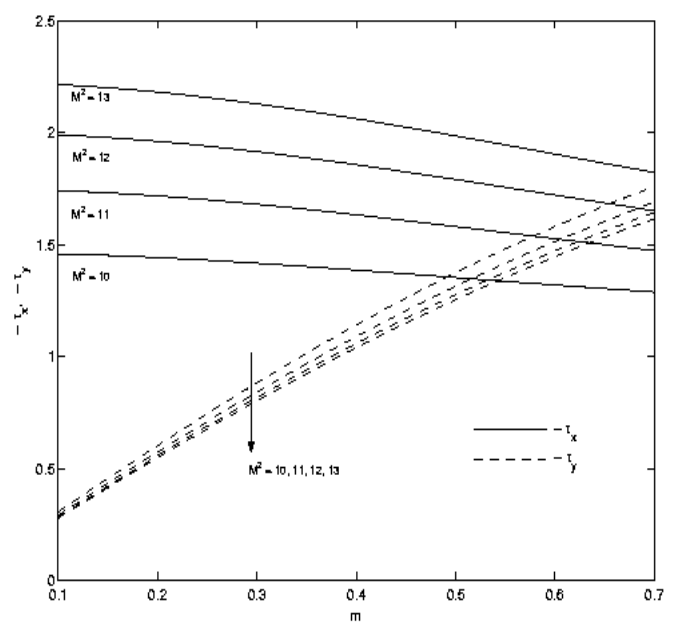

Fig. 11. Shear stresses $\tau_{x}$ and $\tau_{y}$ for $M^{2}$ when $R=4, P r=0.71, G r=5$ and $\tau=0.5$ 


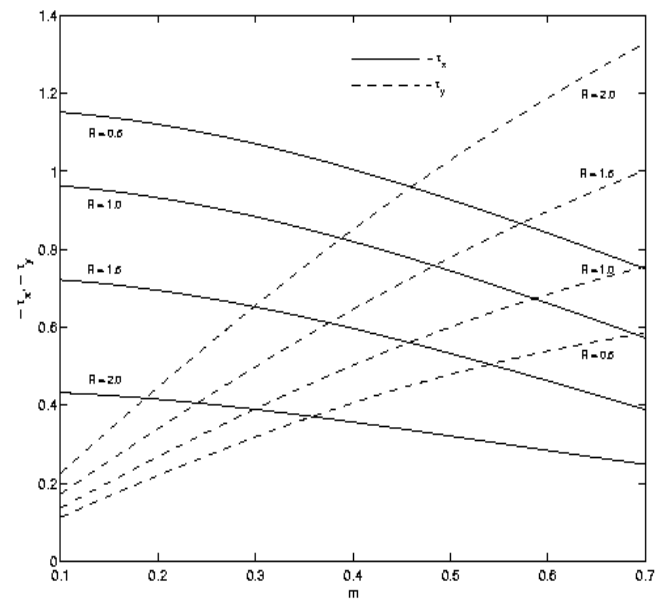

Fig. 12. Shear stresses $\tau_{x}$ and $\tau_{y}$ for $R$ when

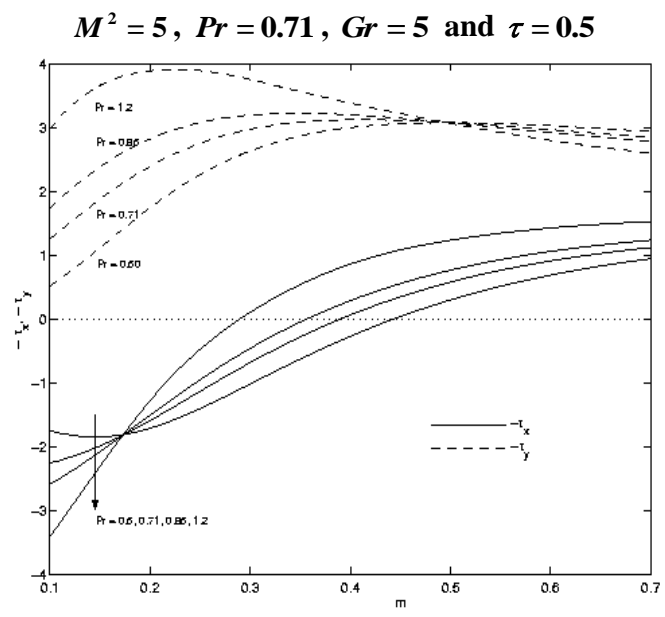

Fig. 13. Shear stresses $\tau_{x}$ and $\tau_{y}$ for $\operatorname{Pr}$ when

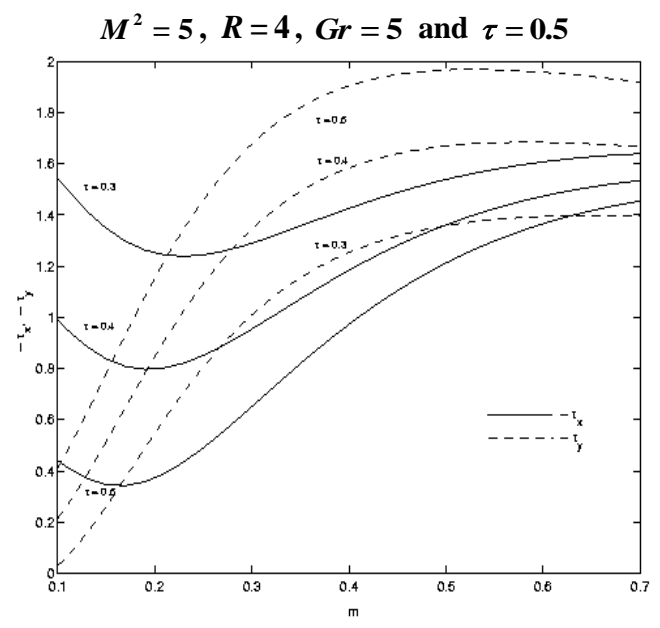

Fig. 14. Shear stresses $\tau_{x}$ and $\tau_{y}$ for $\tau$ when $M^{2}=5, R=4, G r=5$ and $\operatorname{Pr}=0.71$

\section{CONCLUSION}

The effects of Hall current and radiation on MHD flow of a viscous incompressible electrically conducting fluid past a moving vertical plate with variable plate temperature in the presence of a uniform transverse magnetic field have been investigated. It is found that the Hall parameter $m$ accelerates the primary velocity $u_{1}$ as well as the magnitude of the secondary velocity $v_{1}$. An increase in radiation parameter $R$ leads to fall in the primary velocity $u_{1}$ as well as the magnitude of the secondary velocity $v_{1}$. Both the primary velocity $u_{1}$ and the magnitude of the secondary velocity $v_{1}$ increase when time $\tau$ is progressed. Further, the absolute value of the shear stress $\tau_{x}$ due to the primary flow at the moving plate $\eta=0$ decreases whereas the absolute value of the shear stress $\tau_{y}$ due to the secondary flow at the moving plate $\eta=0$ increases with an increase in Hall parameter $m$. The rate of heat transfer $-\left(\frac{\partial \theta}{\partial \eta}\right)_{\eta=0}$ increases with an increase in either Prandtl number $\operatorname{Pr}$ or radiation parameter $R$ or time $\tau$.

\section{ACKNOWLEDGEMENTS}

The authors wish to convey their thanks to the reviewers for very useful comments and suggestions in improving the paper.

\section{REFERENCES}

Aboeldahab, E. M. and M. A. El Aziz (2005). Viscous dissipation and Joule heating effects on MHD free convection from a vertical plate with power-law variation in surface temperature in the presence of Hall and ionslip currents. Applied Mathematical Modelling 29(6), 579 -595.

Aboeldahab, E. M. and E. M. E. Elbarbary (2001). Hall current effect on magnetohydrodynamic free-convection flow past a semiinfinite vertical plate with mass transfer. Int. J. Engineering Science 39(14), 1641-1652.

Alagoa, K. D., G. Tay and T. M. Abbey (1998). Radiative and free convective effects of MHD flow through a porous medium between infinite parallel plates with time-dependent suction. Astrophysics and Space Science 260(4), 455-468.

Aurangzaib, Sharidan Shafie (2011). Effects of Soret and Dufour on unsteady MHD flow by mixed convection over a vertical surface in porous media with internal heat generation, chemical reaction and Hall current. Canadian J. Sci. Engng. Math. 2(4),153-162.

Chaudhary, D., H. Singh and N.C. Jain (2013). Effects of Hall current and thermal radiation on an unsteady free convection slip flow along a vertical plate embedded in a porous medium with constant heat and mass flux. Applied Mathematics and Physics 1(2), 11-26.

Chaudhary, R. C. and P. Jain(2007). Hall effect on MHD mixed convection flow of a viscous elastic fluid past an infinite vertical porous 
plate with mass transfer and radiation, UUR $J$. Phys. 52(10), 110-127.

Choudhury, M. and G. C. Hazarika (2013). The effects of variable viscosity and thermal conductivity on MHD oscillatory free convective flow pasta vertical plate in slip flow regime with variable suction and periodic plate temperature. Journal of Applied Fluid Mechanics 6(2), 277-283.

Cogley, A. C. L., W. G. Vincenti and E. S. Gilles (1968). Differential approximation for radiative heat transfer in a non grey gas near equilibrium. Am. Inst. Aeronat. Astronaut. J. 6, 551-553.

Cookey, C. A., A. Ogolu and V. B. Omubopepple (2003). Influence of viscous dissipation and radiation on unsteady MHD free convection flow past an infinite heated vertical plate in a porous medium with time dependent suction, Int. J. Heat and Mass Transfer 13, 2305-2311.

Cowling, T. G. (1957). Magnetohydrodynamics, Interscience Publisher, Inc, New York.

Helliwell, J. B. and M. F. Mosa (1979). Radiative heat transfer in horizontal magnetohydrodynamic channel flow with buoyancy effects and an axial temperature gradient, Int. J. Heat Mass Transfer 22, 657668 .

Israel-Cookey, C., Amos, E. and C. Nwaigwe (2010). MHD oscillatory Couette flow of a radiating viscous fluid in a porous medium with periodic wall temperature. Ameri.J Sci. Indust.Res. 1(2), 326-331.

Jain, N. C. and H. Singh (2012). Hall and thermal radiation effects on an unsteady rotating free convection slip flow along a porous vertical moving plate. Int. J. of Appl. Mech. Eng. 17 (1), 53-70.

Kinyanjui, M., Kwanza, J. K. and S. M. Uppal (2001). Magnetohydrodynamic free convection heat and mass transfer of a heat generating fluid past an impulsively started infinite vertical porous plate with Hall current and radiation absorption. Energy Conversion and Management 42(8), 917-931.

Ram, P. C. and H. S. Takhar(1994). Unsteady MHD flow with a suspension of spherical particles through a channel. Int. J. Non-Linear Mechanics 29, 775-780.

Reddy, T. S., M. C. Raju and S. V. K. Varma (2013). Unsteady MHD radiative and chemically reactive free convection flow near a moving vertical plate in porous medium. Journal of Applied Fluid Mechanics 6(3), 443451.

Samad, M. A. and M.M. Rahman (2006). Thermal radiation interaction with unsteady MHD flow past a vertical porous plate immersed in a porous medium. J. Naval Architecture and Marine Engineering 3, 7-14.

Shateyi, S., S. S. Mosta and P. Sibanda (2010). The effects of thermal radiation, Hall currents, Soret and Dufour on MHD flow by mixed convection over a vertical surface in porous media, Mathematical Problems in Engineering, 2010.

Seth, G. S., R. Nandkeolyar and M. S. Ansari(2013). Effects of thermal radiation and rotation on unsteady hydromagnetic free convection flow past an impulsively moving vertical plate with ramped temperature in a porous medium. Journal of Applied Fluid Mechanics 6 (1), 27-38.

Shereliff, J. A. (1965). A text book of magnetohydrodynamic, 1 st ed. London: Pergamon Press.

Shit, G. C. and R. Haldar (2010). Combined effects of thermal radiation and Hall current on MHD free-convective flow and mass transfer over a stretching sheet with variable viscosity, Physics Flu-dyn, ar XIv: 1008.0165v1.

Singh, K. D., R. Pathak (2012). Effect of rotation and Hall current on mixed convection MHD flow through a porous medium in a vertical channel in presence of thermal radiation, Indian J. Pure and Appl.Phys., 50, 77-85.

Soundalgekar, V. M. and H. S. Takhar (1977). MHD oscillatory flow past a semi-infinite plate, Am. Inst. Aeronat. Astronaut. J. 15, 457-458.

Soundalgekar, V. M., Patil, M.R. and H.S. Takhar (1981). MHD flow past a vertical oscillating plate, Nuclear Engineering and Design. 64, 43-48.

Takhar, H. S. and G. Nath (1998). Unsteady flow over a stretching surface with a magnetic field in a rotating fluid. Zeitschrift fur Angewwandte Mathematik und Physik 49, 989-1001.

Takhar, H. S. and P. C. $\operatorname{Ram}(1991)$. Free convection in hydromagnetic flows of a viscous heat-generating fluid with wall temperature oscillation and Hall currents. Astrophysics and Space Science 183, 193-198. 